\title{
The Effects of Advertising Copy on Sensory Thoughts and Perceived Taste
}

\author{
RYAN S. ELDER \\ ARADHNA KRISHNA*
}

\begin{abstract}
We propose that advertisement (ad) content for food products can affect taste perception by affecting sensory cognitions. Specifically, we show that multisensory ads result in higher taste perceptions than ads focusing on taste alone, with this result being mediated by the excess of positive over negative sensory thoughts. Since the ad effect is thoughts-driven or cognitive, restricting cognitive resources (imposing cognitive load) attenuates the enhancing effect of the multiple-sense ad. Our results are exhibited across three experiments and have many implications for cognition and sensory perception research within consumer behavior, as well as several practical implications.
\end{abstract}

$\mathrm{F}_{\mathrm{s}}$ ood advertising is big business. Kraft Foods spent \$1.5 billion in 2007 on advertising in the United States alone, whereas PepsiCo spent \$1.31 billion and McDonalds spent $\$ 1.14$ billion (Advertising Age Data Center 2008). The financial importance of this domain raises the question, how should one advertise for food? A quick glance at current ads shows the obvious: mention the taste of the food. This is expected since the ad is for food after all. The less obvious and consequently seldom used solution is to bring attention to the unique multisensory aspects of taste perception. In this research, we suggest how and why multisensory advertising for food ads can enhance taste perceptions.

In this article we explore whether other senses are so physiologically closely tied to taste that mentioning them will make no difference and whether an ad in general can have an impact on taste perceptions. By exploring if the ad can affect taste itself, we test an additional possible effect of the ad. While food advertising is typically used to spark interest in the food or an intention to buy it, it is not usually used for affecting taste perception. Further, if the ad does affect taste, then we are also suggesting that taste is affected by cognition and is not automatically incorporated into perceptions. Besides looking at the effect of ads on taste perception,

*Ryan S. Elder (rselder@umich.edu) is a doctoral candidate in marketing and Aradhna Krishna (aradhna@umich.edu) is the Isadore and Leon Winkelman Professor of Marketing at the Ross School of Business, University of Michigan, 701 Tappan Street, Ann Arbor, MI 48109-1234. Author names are in alphabetical order. The authors thank Daphna Oyserman, participants at the Michigan Marketing lunch seminars, the editor, the associate editor, and the three reviewers for comments on this article at various stages.

John Deighton served as editor and Stephen Hoch served as associate editor for this article.

Electronically published June 25, 2009 we additionally explore what happens to consumers' thoughts about the food (how they change) when the ad is changed. We also examine if these thoughts drive the change in taste perception.

This research has both theoretical and substantive implications. First, we show that ads can affect sensory perceptions and that sensory thoughts mediate the affect of ads on perception. More specifically, we show that ads mentioning senses other than taste can increase positive sensory thoughts about the food and consequently taste. Second, we show that the processing of ads is deliberate and cognitive, so that the enhancing effect of multiple-sense ads is reduced when cognitive resources are constrained. Our hypotheses are supported across three experiments.

Our research has many practical implications for ad executives and managers since it can easily and readily be applied in directing ad copy for food products. In the rest of the article, we build our conceptual framework and hypotheses, elaborate on the experiments, and finally present conclusions and ideas for future research.

\section{LITERATURE REVIEW AND HYPOTHESES}

One of the main focal points of this article is to explore the interaction of cognition and sensory perception, particularly taste. Taste is a curiously unique sensation as it is composed not only of one sensory input (i.e., from the tongue) but is also created by incorporating multiple sensory inputs. As such, taste is suggestible and ambiguous. We propose that, in addition to a reliance on intrinsic cues from the food itself, taste is susceptible to extrinsic cues such as advertising.

We begin our literature review with a brief overview of the neuroscience and physiology literature addressing the in- 
trinsic multisensory composition of taste. We then focus on the more critical dimension of taste perception to our research: the impact of extrinsic cues such as advertising.

\section{Effect of Intrinsic Cues on Taste Perception-What Is Taste Perception?}

When we think of taste perception, we immediately think of sensations on the tongue. However, despite our seemingly constant exposure to food, we have remarkable difficulty in discerning one taste from another with just our taste buds. Part of this ineptitude stems from the limited number of distinct tastes that we can detect. Until recently, our taste buds were known to detect only sweet, sour, salty, and bitter tastes. A new taste, umami, discovered in 1909 (Ikeda 2002), only recently received neurophysiological support for its existence as a distinct taste receptor (Chaudhari, Landin, and Roper 2000). Unfortunately, even with the addition of this fifth taste, it is still difficult to accurately judge the complex sensation of taste. However, taste is not physiologically made up of sensations from taste buds only; it also relies heavily on input from the other senses.

Imagine eating a handful of popcorn. It is impossible to simply focus on the sensations of your tongue. Does not the mouth-watering smell of the butter, the feel of the popcorn in your hands and mouth, the popcorn's warmth, the way it sounds when you chew it, and the popcorn's visual appearance all lead to an overall multisensory taste experience? The fact that every sense has some role in generating taste has, in fact, recently received neurophysiological support (Rolls 2005; Small and Jones-Gotman 2001). Rolls shows that the pure effects of gustatory stimuli are represented in the primary taste cortex (frontal operculum/insula), whereas the convergence of multiple sensory inputs used to represent taste occurs in part of the orbitofrontal cortex, referred to as the secondary taste cortex (Rolls 2005).

The primary accompanying sense for taste is olfaction, or how the food smells (Small and Prescott 2005). In fact, smell affects taste both before (orthonasal) and after (retronasal) food enters our mouth (Rozin 1982). Smell plays such an integral role in taste perception that, without it, it is difficult to distinguish a potato from an apple or wine from apple juice (Herz 2007). The intrinsic visual appearance of the food also contributes to the sense of taste in generating expectations and perceptions of flavor (Dubose, Cardello, and Maller 1980) and can ultimately dominate gustatory cues altogether (Hoegg and Alba 2007). The sound the food makes when bitten plays a key role in taste perceptions for certain food items (e.g., potato chips, celery, crackers), affecting perceived freshness as well as perceived quality (Zampini and Spence 2004). Relatedly, the texture (de Araujo and Rolls 2004) and temperature of food can affect taste. Recent research has shown that temperature sensations on the tongue are directly related to taste. Specifically, warming the tongue elicits sweet and bitter tastes, whereas cooling the tongue leads to sour and salty taste perceptions (Cruz and Green 2000).
It is thus evident from the physiology and neuroscience literature that taste is derived from multiple intrinsic sensory components, including smell, vision, sound, and touch (including texture and temperature). However, the automaticity of these inputs is largely unexplored, leaving the question as to whether or not advertising incorporating these cues will have an impact on taste perceptions. This question addresses the automaticity of intrinsic cues, as well as the general impact of extrinsic cues, such as advertising, on taste perceptions. We next focus on relevant literature within consumer behavior and psychology addressing the impact of extrinsic cues on taste perceptions and formally present our hypotheses.

\section{Effect of Extrinsic Cues on Taste Perception}

Let us distinguish first between a more deliberate topdown process for taste perception versus a more automatic bottom-up process. Top-down processing holds that external information provided about the food is processed more deliberately and that it affects taste perception in a cognitive manner, whereas bottom-up processing would suggest that information about the food is processed more automatically and heuristically, driven by inherent aspects of the stimulus such as the intrinsic cues discussed earlier (Smith and Kosslyn 2007). Sometimes it is questionable which process is working. Also, both processes may operate simultaneously and interact with one another. One instance where top-down processes have a large impact on perceptions is with ambiguous or suggestible experiences (Hoch and Ha 1986). Within the present context, the ambiguity of a taste experience would then lead to more susceptibility to and increased utilization of external influences in forming overall taste perceptions. We look at such outside influences both in more bottom-up and more top-down processing contexts.

Research examining more automatic, bottom-up processing effects in taste perceptions includes Raghunathan, Naylor, and Hoyer (2006), which examines the effect of labeling a food item as either healthy or unhealthy. The authors receive support for an unhealthy-equals-tasty intuition that consumers hold, whereby food categorized as unhealthy results in higher taste perceptions than food categorized as healthy. They provide support for an automatic, bottom-up process by showing that, in an implicit association test (IAT), individuals are quicker to categorize unhealthy (vs. healthy) foods as tasty. Krishna and Morrin (2008) demonstrate the automatic effect of another extrinsic cue, product haptics, on taste perception. In multiple experiments, they show that the haptic quality of glasses from which water and other drinks are consumed can affect taste perception. They argue that the haptic effect on taste is automatic and that more deliberate processing would make people realize that the containers are nondiagnostic for taste and should not affect their perception. Thus, even extrinsic cues can operate in a more automatic manner in influencing taste perceptions.

Moving to more controlled top-down processing, which is the proposed mechanism for the effects in the present research, Allison and Uhl (1964) explore the impact of brand 
name on subsequent taste preferences. The authors administer a blind taste test of beers (by removing identifying labels) to experienced beer drinkers and find that participants cannot correctly discriminate between the beers. However, when the beers are labeled, the participants rate their favorite beer higher than the others. This would be considered top-down deliberate processing, with people linking their preferred brand name with the better-tasting beer. Lee, Frederick, and Ariely (2006) additionally show that such extrinsic cues not only alter preferences among beers but also can change one's taste experience altogether. In their studies, the stated ingredients (whether the beer had balsamic vinegar or not) affect the taste experience. Levin and Gaeth (1988) show that the specific verbalization of fat amount in meat (e.g., $75 \%$ lean or $25 \%$ fat) affects the perceived leanness and taste of the meat. Recently, Hoegg and Alba (2007) show the impact of several extrinsic cues for orange juice, including brand name, price, and region of origin, on taste discrimination and taste preference. Their findings include a national brand receiving better taste evaluations than a store brand and differences in the color of orange juice leading to greater perceived taste differences than differences in brand labels. This could again be top-down processing, with people expecting, for instance, that a national brand uses better oranges and should have better orange juice.

Top-down deliberate processing of ads, with the ad content affecting initial perceptions, has been shown in prior research comparing verbal and visual ads. Famously, Mitchell and Olson (1981) show that a verbal ad (Brand I Facial tissues are soft) results in the tissues being perceived to be less soft than a visual ad (picture of a kitten). Edell and Staelin (1983, 46) suggest that the verbal message of the ad is processed more cognitively and can guide the processing of the picture contained within the ad. Our research contributes to prior research on the impact of cognitions on consumer behavior in that we are subjecting participants to an actual consumption experience where they will evaluate a sensation. In prior research, such as Mitchell and Olson (1981) and Edell and Staelin (1983), subjects simply judged the product by looking at the ad. Nonetheless, this research suggests that the content of ads can be processed cognitively and could affect taste perception by framing the overall experience.

Prior research also demonstrates that the ad can guide the types of thoughts generated. Using verbal protocols, Edell and Staelin (1983) find that an objective ad (e.g., car is fourwheel drive) results in fewer support arguments and more counterarguments than a subjective ad (car drives well on snow). For food ads, a multiple-sense ad should direct sensory thoughts that can be about all five senses to be more positive compared to the single-sense ad that focuses on taste alone. The ad can explicitly mention the niceties of all five senses. However, even if it does not-for instance, if we consider just an ad slogan like "taste is all five senses"- the mere fact that the ad is mentioning all five senses is suggestive that the food rates high on all five senses; therefore the ad should direct thoughts for all sensory modalities to be positive.
The literature presented on the effect of extrinsic cues on taste perception should work in concert with the literature on the physiological composition of taste. We propose that the cognitions generated by an extrinsic cue, such as an ad, will have an impact on sensations and ultimately affect taste perceptions. As taste is composed of all five senses, thoughts about all five senses should affect taste. Ads mentioning all five senses would be more likely to direct thoughts about these senses to be positive compared to ads that talk about taste alone. Additionally, perceived taste should be better the more positive sensory thoughts one has and be worse the more negative sensory thoughts one has. Hence, the surplus of positive over negative thoughts should drive taste perception.

With the effects depending largely on thought generation and cognition, we anticipate the availability of cognitive resources to affect the ad taste effects. Specifically, with topdown or largely cognitive processing, the introduction of cognitive load should distract attention away from the ad (Nowlis and Shiv 2005; Shiv and Nowlis 2004) and attenuate the effect that the ad has on taste perceptions.

More formally, we propose that:

H1: Perceived taste for food will be better with ads that mention multiple senses (multiple-sense ads) compared to ads that mention taste alone (singlesense ads).

H2a: There will be more positive sensory thoughts with a multiple- versus a single-sense ad.

H2b: The number of positive minus negative sensory thoughts will mediate the effect of an ad on perceived taste.

H3: Cognitive load will reduce the effect of a multiple-sense versus single-sense ad on the number of positive thoughts for food and on taste perceptions.

We test our set of hypotheses in a series of three experiments, using different foods as stimuli (chewing gum, potato chips, and popcorn) to test for the robustness of our results.

\section{STUDY 1: AD SLOGAN, SENSORY THOUGHTS, AND TASTE (GUM STUDY)}

In this study, we test if multiple-sense ads result in higher perceived taste compared to single-sense ads (hypothesis 1) and in more positive thoughts compared to single-sense ads (hypothesis 2a) and if sensory thoughts mediate the effect of ads on perceived taste (hypothesis $2 b$ ). In order to conduct a strong initial test of our process, we use very simple ad slogans, with one focusing explicitly on taste ("Long Lasting Flavor") and the other on the general sensory experience ("Stimulate Your Senses"). We posit that, even at this very general level, drawing attention to sensory experiences beyond taste will result in more sensory thoughts and consequently better taste perceptions. 
TABLE 1

STUDY 1: GUM THOUGHT MEANS BY SLOGAN CONDITION

\begin{tabular}{|c|c|c|c|c|c|c|c|c|c|c|}
\hline Condition & $\begin{array}{c}\text { Positive } \\
\text { minus } \\
\text { negative }\end{array}$ & $\begin{array}{c}\text { Total } \\
\text { sensory }\end{array}$ & Positive & Negative & Neutral & Taste & Smell & Sight & Texture & Sound \\
\hline Multiple sense & 1.29 & 2.64 & 1.82 & .54 & .29 & 1.46 & .50 & .18 & .50 & .00 \\
\hline Single sense & .38 & 1.81 & .92 & .54 & .42 & 1.08 & .31 & .08 & .35 & .00 \\
\hline Total & .85 & .24 & 1.39 & .54 & .35 & 1.28 & .41 & .13 & .43 & .00 \\
\hline
\end{tabular}

\section{Method}

Pretest. Pretests were conducted with 27 participants recruited from the business school lounge at the University of Michigan. Each participant filled out a brief questionnaire that contained one of two ad slogans. One questionnaire contained the multiple-sense slogan ("Stimulate Your Senses"), whereas the other contained the single-sense slogan ("Long Lasting Flavor"). Participants were asked to give their overall evaluations of the slogans on three separate dimensions (7-point scales anchored at $1=\mathrm{bad} /$ unfavorable/dislike and $7=$ good/favorable/like). These three items were combined to form the attitude toward the ad scale $(\alpha=.94$; Mitchell and Olson 1981). We then compared the means of the scale for both slogans and found no significant difference $\left(M_{\text {multiple }}=4.90, n=13, M_{\text {single }}\right.$ $=4.73, n=14 ; p>.5)$. It should also be noted that the number of words in the multiple-sense and single-sense slogans were intentionally kept the same.

Design and Procedure. Fifty-four undergraduates at the University of Michigan participated in groups, with a maximum size of 10, in exchange for course credit. Each participant was randomly assigned to one of the two conditions (single-sense ad or multiple-sense ad), placed in front of a folder, and presented with a cover sheet with the following instructions:

A food distributor has recently created a new line of chewing gum which is being test marketed in several areas across the country. You will have the opportunity of trying this gum today. Below is the tagline for the gum:

Participants then read one of the two slogans and were told to ask the experimenter for the piece of gum when done reading. The gum was the peppermint flavor of Wrigley's Extra brand, and it was served to the participants on a plate with the packaging removed. Before turning the page to answer the questions, participants were instructed to chew the gum. Then, while chewing, participants were asked to write down any thoughts that came to their mind. Note that the multiple-sense slogan did not list any specific senses that would be readily available to participants when listing their thoughts. Following the thought-listing activity, participants rated the taste of the gum on a 7-point scale $(1=$ very poor taste, $7=$ very good taste $)$. Upon completion of the questionnaire, participants were given a debriefing report and dismissed.

\section{Results and Discussion}

Results. An ANOVA on taste perceptions revealed the hypothesized effect of single- versus multiple-sense slogans on perceived taste $\left(M_{\text {multiple }}=5.39, M_{\text {single }}=4.77 ; F(1,52)\right.$ $=6.60, p<.05)$, with the slogan "Stimulate Your Senses" leading to higher taste perceptions than the slogan "Long Lasting Flavor." This result provides support for hypothesis 1 , whereby multiple-sense advertising leads to higher taste perceptions than single-sense advertising.

Participants' thoughts were coded for valence as positive (e.g., "It's good and flavorful"), negative (e.g., "I don't really like the flavor"), and neutral (e.g., "It's minty"). The thoughts were further coded by content as being primarily sensory (e.g., "I like the texture"), brand related (e.g., "I prefer Orbit gum"), or slogan related (e.g., "Not very stimulating"). Thoughts were rated by two independent coders, and intercoder reliability was $91.3 \%$; disagreements were resolved through discussion. Table 1 presents the average number of thoughts by condition.

Participants listed a few thoughts on the slogan (.04 per subject for both single-sense and multiple-sense ads) and the brand ( 0.19 and 0.18 per subject for the single- and multiplesense slogans, respectively), but the vast majority of listed thoughts were sensory in nature (1.81 and 2.64 for the singlesense and multiple-sense slogans, respectively). The mean number of total sensory thoughts was significantly higher for the multiple-sense slogan $(M=2.64)$ than for the singlesense slogan $(M=1.81 ; F(1,52)=5.66, p<.05)$. Within the total sensory thoughts, there were also more positive thoughts in the multiple-sense condition than in the singlesense condition $\left(M_{\text {multiple }}=1.82, M_{\text {single }}=.92 ; F(1,52)=\right.$ $7.65, p<.01$ ), supporting hypothesis 2 a. Proportionally, most of the sensory thoughts were positive, and there was a significantly higher proportion of positive sensory thoughts in the multiple-sense condition $(69 \%)$ versus the single-sense $(51 \%)$ condition $(z=1.97, p<.05)$. There were no significant differences between negative and neutral proportions. As shown in table 1, there were directionally more thoughts concerning smell, taste, vision, and feel (haptics) for the multiple-sense ad versus the single-sense ad. We are most interested, however, in the balance of positive over negative sensory thoughts. This measure captures the overall valence of 
the participant's sensory thoughts, which we anticipate drives the effect of the slogan on taste perceptions. The number and the type of cognitive verbal responses have also been used in earlier research as measures of process (Cacioppo and Petty 1981). Consistent with this reasoning, we find that the net thoughts are significantly more positive in the multiple-sense slogan condition than in the single-sense slogan condition $(1.29$ vs. $0.38 ; F(1,52)=4.75, p<.05)$.

Mediation tests involved additional analyses to determine if the effect of the slogan on taste perceptions was mediated by the number of net positive sensory thoughts. The first criterion of mediation was met (Baron and Kenny 1986), as the ANOVA reported earlier shows a significant effect of slogan on perceived taste $(F(1,52)=6.60, p<.05)$. We also received support for the second criterion of mediation by showing a significant relationship between the slogan condition and net positive sensory thoughts $(F(1,52)=$ $4.75, p<.05)$. An ANCOVA run with perceived taste as the dependent variable, slogan as the independent variable, and net positive sensory thoughts as the covariate provides support for the third criterion of mediation. The initial significant main effect of slogan on perceived taste is now only marginally significant $(F(1,51)=3.15, p=.082)$, whereas the net number of positive sensory thoughts has a significant effect on perceived taste $(F(1,51)=8.94, p<.01)$. As indicated by the Sobel (1982) test $(z=1.8, p<.08)$, these results are suggestive of the net positive sensory thoughts mediating the effect of ad slogans on perceived taste.

Discussion. In study 1, we obtain support for hypothesis 1 , finding that multiple-sense ads result in better taste perception versus single-sense ads. Multiple-sense ads also result in significantly more net positive sensory thoughts than single-sense ads, supporting hypothesis $2 \mathrm{a}$. Further, the number of net positive sensory thoughts mediates the effects of the slogan on taste perceptions, supporting hypothesis $2 b$. Study 2 addresses two limitations of study 1, namely, the single-item dependent variable and the general ambiguity of the slogans used in study 1 , which could affect the perceived amount of informativeness contained in the slogans. Addressing these limitations will help to more fully explicate the theoretical and practical implications of our research. In study 2 , we also use potato chips to test the robustness of the effects obtained in study 1 .

\section{STUDY 2: ADS, SENSORY THOUGHTS, AND TASTE (POTATO CHIPS STUDY)}

We created two ads for study 2 . One of these ads described different sensory experiences (taste, smell, texture) when eating potato chips, whereas the other ad described an equal number of taste experiences when eating potato chips. The single-sense (multiple-sense) ad read:

Our potato chips deliver the taste you crave. From the first bite you'll savor the rich barbecue flavor (smell) and enjoy the delicious salty taste (crunchy texture)—our potato chips are the perfect choice for your snacking.
To further ensure that the multiple-sense slogan did not have more perceived information than the single-sense slogan, we carefully pretested the ads.

\section{Method}

Pretest. The two ads were pretested on complexity and informativeness, as well as on other standard attitudes toward the ad measures. The pretests were conducted with 46 University of Michigan undergraduate students enrolled in an introductory marketing course, and they employed a betweensubjects design. After reading the ad, participants answered a series of questions regarding their opinions and attitudes toward the ad. Participants rated the degree to which they agreed or disagreed with two statements about the ad ("The ad was informative/complex"; 1 = strongly disagree, $7=$ strongly agree). We also captured overall evaluations of the ads on three separate dimensions (7-point scales anchored at $1=\mathrm{bad} /$ unfavorable/dislike and $7=\operatorname{good} /$ favorable/like; Mitchell and Olson 1981). These three items were combined to form an attitude toward the ad scale $(\alpha=.83)$.

Evaluations of the ads did not differ across the three dimensions (all $p>.35$ ). The ads were perceived equal on informativeness $\left(M_{\text {single }}=4.61, M_{\text {multiple }}=4.74 ; p>.5\right)$, complexity $\left(M_{\text {single }}=2.91, M_{\text {multiple }}=2.57 ; p>.35\right)$, and overall attitude toward the ad $(p>.5)$.

Design and Procedure. The experiment used a onefactor between-subjects design, with the ad (multiple-sense or single-sense) serving as the manipulated factor. We chose barbecue flavored, kettle-cooked potato chips to be the food eaten during the experiment. Ninety-two undergraduate students at the University of Michigan participated in the study in exchange for course credit, doing so in groups with a maximum size of 10. Participants were first introduced to the project with a page describing the purpose of the research, which was to evaluate ads and products that either currently exist on the market or are in a testing phase. Participants then read the ad for the potato chips. Upon reading the ad, participants were instructed to raise their hands to ask the experimenter for the potato chips. The experimenter then placed a plain white cup of chips with a napkin in front of the participant. Participants then ate the chips before moving on in the questionnaire. All instructions were contained in the questionnaire, including when to ask the experimenter for the food item, and thus participants performed the experiment's activities self-paced.

Measures. Participants began the questionnaire by listing any thoughts they had while eating the chips. Then they were asked to evaluate the chips on three dimensions: the overall quality $(1=$ very poor quality, $9=$ very good quality), the overall taste $(1=$ very poor taste, $9=$ very good taste), and how delicious the potato chips were $(1=$ not at all delicious, $9=$ very delicious). 


\section{Results and Discussion}

Results. We conducted an ANOVA with the three taste measures combined into one scale as the dependent measure $(\alpha=.92)$ and ad as the independent variable. We found support for the hypothesized effect of ad on taste (hypothesis 1), with the multiple-sense ad leading to significantly higher taste perceptions than the single-sense ad $\left(M_{\text {multiple }}=6.78, M_{\text {single }}\right.$ $=5.67 ; F(1,90)=9.56, p<.005)$.

We posited that sensory thoughts would mediate the effects of ad on taste perceptions (hypothesis 2b). Cognitive responses were coded in an identical manner to study 1 . Again, we find more positive sensory thoughts in the multiple-sense condition $(M=1.98)$ than in the single-sense condition $(M=1.25 ; F(1,90)=8.90, p<.005)$, supporting hypothesis $2 \mathrm{a}$. The proportion of the positive sensory thoughts was again significantly greater in the multiple-sense condition $(68 \%)$ versus the single-sense $(40 \%)$ condition $(z=4.49, p<.01)$. In addition, the net positive sensory thoughts were greater in the multiple-sense condition $(M=1.70)$ than in the single-sense condition $(M=0.29$; $F(1,90)=20.44, p<.001)$. We conducted additional analyses to check for mediation. As reported, the first two criteria for mediation were met, with the ad having a significant effect on both taste perceptions and net positive sensory thoughts. An ANCOVA was run to check for the third criterion of mediation, with perceived taste as the dependent variable, ad as the independent variable, and net positive sensory thoughts as the covariate. The initial significant impact of ad on taste perceptions is no longer significant $(F(1,89)=.17, p>.5)$, while the net number of positive sensory thoughts has a significant effect on perceived taste $(F(1,89)=50.81, p<.001)$. Thus net positive sensory thoughts again mediate the effect of ad on taste perceptions, as indicated by the Sobel (1982) test $(z=2.8, p<.01)$. These results add support further to hypothesis $2 \mathrm{~b}$.

Discussion. The findings of study 2 provide corroborating support for the process involved in the effect of advertising on taste perceptions. The multiple-sense ad was effective in generating more positive sensory thoughts and better taste perceptions, supporting hypotheses 1 and hypothesis $2 \mathrm{a}$. Further, the number of net positive sensory thoughts was found to mediate the effects of the ads on taste perceptions, supporting hypothesis $2 \mathrm{~b}$.

A remaining question, however, is to what extent these sensory effects depend on effortful, deliberative processing. The effectiveness of the ads in the prior studies was mediated by sensory cognitions. Therefore, would the effects of the ads obtain if participants were limited in their cognitive capacity? In study 3, we address any moderating role of cognitive load on the ad-taste effect.

\section{STUDY 3: COGNITIVE LOAD AS A MODERATOR OF THE AD-TASTE EFFECT (POPCORN STUDY)}

The effects of ads on taste perceptions in the prior studies are mediated by sensory thoughts. These results are con- sistent with our theoretical framework that the effects are largely cognitive or top-down in nature. Therefore, we anticipate the introduction of cognitive load to attenuate our effects, leading to less dissimilar taste perceptions across ad conditions (hypothesis 3). Put another way, we are arguing that the multiple-sense ad (vs. a single-sense ad) has a smaller effect on enhancing taste perceptions under the condition of cognitive load.

Initially, this hypothesis may seem at odds with results from recent research on in-store sampling and distraction (Nowlis and Shiv 2005; Shiv and Nowlis 2004). The main finding across these articles is that distracting consumers (imposing cognitive load) while taste testing will lead to a heightened focus on the affective experience versus any informational input; this increases the subsequent likelihood of choosing a more affective product from a set of products (e.g., milk chocolate vs. soy chocolate) and also increases consumption pleasure. At a superficial level, we seem to be arguing that load will decrease and not increase consumption pleasure from food as these studies have found. However, note that, while ads are informational, the ads in our experiments lead to affective (sensory) thoughts, so distraction from the ad is a distraction from affective consequences. As the results from our prior two studies show, the multiplesense ads we employ lead to affectively valenced consequences (i.e., more positive sensory thoughts and heightened taste perceptions). Hence, we argue that a distraction from the ads will reduce the taste-enhancing effect of the multiplesense ad. We now test hypothesis 3 .

\section{Method}

Design. A 2 (ad: multiple-sense or single-sense) $\times 2$ (cognitive load: yes or no) between-subjects full factorial design was used in study 3 . One hundred and twelve University of Michigan undergraduates participated in the experiment as part of a subject pool. We used popcorn as the food product in this study. The brand name for the popcorn, Emerald Aisle, was fictitious in order to limit confounds related to prior brand exposure. The ads below were used to describe the popcorn in the single-sense (multiple-sense) conditions:

Emerald Aisle popcorn delivers the taste (smell) of a movie theater in your own home. You'll taste (see) the perfect amount of butter and salt in every handful. With its delicious, buttery flavor (texture) and a taste that dances on your tongue (crunch that's music to your ears), Emerald Aisle popcorn is the perfect choice for all your snacking.

Pretest. The two ads were pretested on 40 participants to ensure equivalence in participants' overall attitudes toward the two ads ( $\alpha=.97$; Mitchell and Olson 1981), informativeness, and complexity, as in the prior pretests. A comparison of the means across ads showed no difference for any of the measures (all $p>.5$ ). 
Procedure. Participants completed the study in groups with an upper limit of 10 people. Before being given the ad, participants in the load condition were given a separate task. In this task, participants were given a sheet that contained the roster for a fifth-grade class. They were instructed to remember which first name went with which last name. Similar tasks have been shown to be cognitively taxing in earlier research (Gilbert, Giesler, and Morris 1995; Gilbert and Hixon 1991). Participants were given 1 minute to examine the class roster, and they then moved to the next questionnaire, which contained the popcorn ad (participants in the no-load condition moved directly to the popcorn questionnaire). To be consistent with the cover story for imposing cognitive load, participants were given a memory test after they completed the questionnaire related to the ad. This test asked two questions on the names of students in the fifth grade class (e.g., "What is Jay's last name?").

The popcorn used was the private-label brand from a local grocery store. Each participant was handed a napkin and a white plastic cup half full of popcorn. The cups of popcorn were prepared before each experimental session, and participants were not exposed to the packaging. Each participant was instructed to eat the popcorn first before moving on to answer the questions. The first question given to participants was to list any thoughts they had while eating the popcorn. Participants then answered questions regarding their perceptions of the taste of the popcorn. Similar to study 2 , participants reported how they perceived the taste $(1=$ very poor taste, $7=$ very good taste $)$, quality $(1=$ very poor quality, $7=$ very good quality), and deliciousness ( 1 $=$ not at all delicious, $7=$ very delicious) of the popcorn. These items were combined to form a three-item scale measuring taste perceptions $(\alpha=.90)$. After completing the questionnaire, participants in the cognitive load condition were given the memory test questions. Participants were also given the opportunity to report any suspicions they had regarding the purpose of the study. No participant correctly guessed or showed insight into the experimental hypotheses.

\section{Results and Discussion}

We conducted an ANOVA with taste perception as the dependent variable and ad and cognitive load as independent variables. There was a significant main effect of ad on taste perceptions, with the multiple-sense ad leading to higher taste perceptions than the single-sense ad $\left(M_{\text {multiple }}=4.14\right.$ vs. $\left.M_{\text {single }}=3.63 ; F(1,108)=6.39, p<.05\right)$, adding further support to hypothesis 1 . This main effect was qualified by the hypothesized two-way interaction of load and ad on overall taste perception $(F(1,108)=7.79, p<.01)$. The main effect of load was not significant $(p>.15)$. Figure 1 graphically presents the cell means of taste perceptions by condition.

Simple effect tests revealed a significant difference between the multiple-sense and single-sense ads in the no-load conditions $\left(M_{\text {multiple }}=4.67, M_{\text {single }}=3.48 ; F(1,108)=13.60\right.$, $p<.01)$, with taste perceptions in the multiple-sense ad con-
FIGURE 1

STUDY 3: MEANS OF PERCEIVED TASTE (POPCORN)

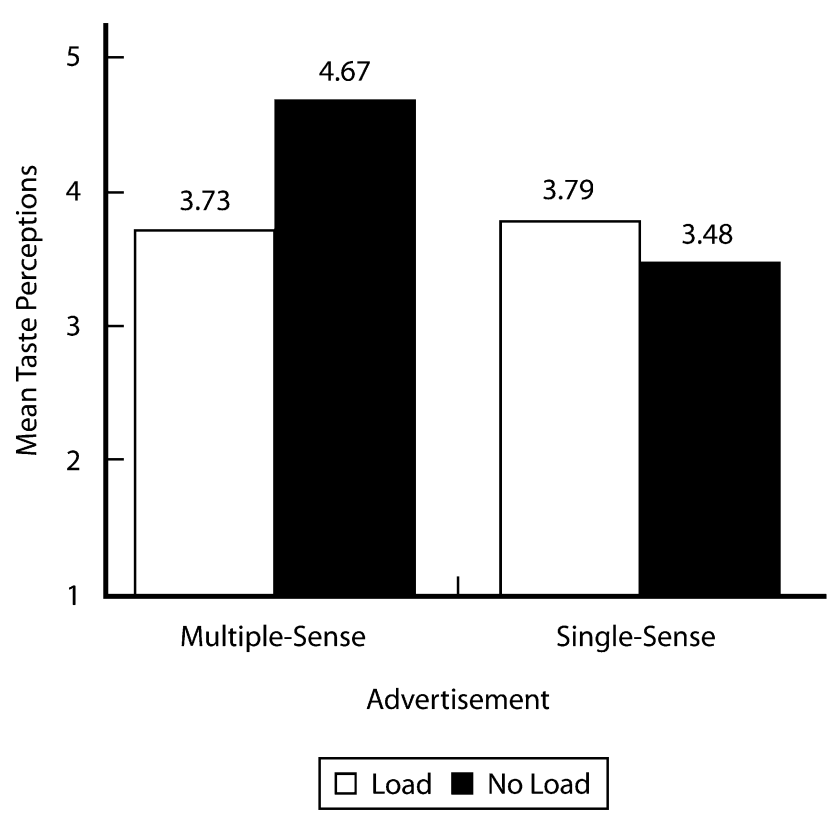

dition being significantly higher; however, there was no significant difference between the multiple-sense and singlesense ads in the load condition $(p>.8)$. This is consistent with hypothesis 3 .

Additional simple effect tests revealed a significant difference between the load and no-load conditions when subjects were exposed to the multiple-sense ad $\left(M_{\text {load }}=3.73\right.$, $\left.M_{\text {noload }}=4.67 ; t(108)=2.92, p<.01\right)$, with taste in the no-load condition being significantly higher. Simple effect tests also showed that the effect of the single-sense ad on perceived taste was not significantly different across the two load conditions $\left(M_{\text {load }}=3.79, M_{\text {noload }}=3.48 ; t(108)\right.$ $=1.00, p>.3$ ).

We also conducted an ANOVA with net positive sensory thoughts as the dependent variable and ad and load as the independent variables. Thoughts followed a similar pattern to taste perceptions, such that there was a significant main effect of ad with the multiple-sense ad leading to more net positive thoughts than the single-sense ad $\left(M_{\text {multiple }}=\right.$ 0.25 vs. $\left.M_{\text {single }}=-0.39 ; F(1,108)=4.80, p<.05\right)$. This main effect was also qualified by the hypothesized twoway interaction of ad and load $(F(1,108)=4.16, p<$ $.05)$.

As in prior studies, we wanted to determine if net positive sensory thoughts mediate the relationship between the independent variables (ad and load) and the dependent variable (taste perceptions). As already shown, the first two criteria of mediation are met as the interaction of ad and load on taste perceptions was significant and the interaction of ad and load on net positive sensory thoughts was also significant. We conducted an ANCOVA to check for the final 
criterion of mediation with ad and load as the independent variables, net positive sensory thoughts as the covariate, and taste perceptions as the dependent variable. The prior significant interaction of ad and load on taste perceptions is no longer significant ( $p=\mathrm{NS}$ ), whereas the net positive sensory thoughts are significant $(F(1,108)=42.03, p<.001)$. A Sobel (1982) test conducted supports mediation $(z=1.95$, $p=.05$ ), again adding support to hypothesis $2 \mathrm{~b}$.

The results of study 3 add further support to our hypotheses, and they also establish potential boundary conditions for our effects. Taste perceptions differed between single-sense and multiple-sense ad conditions only when cognitive resources were available. That is, when participants could appropriate an ample amount of cognitive resources to the multiple-sense $\mathrm{ad}$, the overall taste perception was better than in the singlesense condition. In the condition where cognitive resources were constrained, there was no difference in perceived taste between the multiple-sense and single-sense ads. This experiment further demonstrates the cognitive nature of the effects and contributes theoretically by showing that the effect of distraction during a consumption experience may be contingent upon the type of information presented.

\section{GENERAL DISCUSSION}

Research on sensory perception within marketing has largely focused on the study of vision (see Krishna [2007] for a review), with the other senses receiving scattered attention; however, this attention is intensifying (see Peck and Childers [2008] for a review). A primary objective of this article is to contribute to the growing literature on sensory perception within marketing in showing that advertising copy for a food product can affect resulting cognitions during consumption and ultimately affect taste perceptions. Through a series of three studies, we showed that multiplesense versus single-sense ads led to heightened taste perceptions, within some boundary conditions.

With study 1 (chewing gum), we showed that a simple slogan could affect taste perceptions. Specifically, we showed that a multiple-sense slogan led to higher taste perceptions than a single-sense slogan. Study 2 (potato chips) replicated and extended these results by showing the effect of verbal sensory advertising on taste perceptions. Study 3 (popcorn) further explicated the deliberate, top-down nature of our results, showing that the effect of the ad on taste perceptions is moderated by cognitive resource availability. Further, studies 1-3 show the mediating effect of net positive sensory thoughts on perceived taste.

Our research makes important contributions to both the consumer behavior and sensory perception literatures. Our contribution to marketing is an explication of the effects of ads on taste perception. This extends the impact of advertising beyond typical evaluation variables such as awareness and purchase intentions. We demonstrate that ads (for food) can have a significant impact on perceived taste. We also contribute to perception research by providing evidence for the impact of verbal stimuli on sensory evaluations. Finally, we make an attempt to bridge the gap between physiology and neuroscience and consumer behavior, showing promising potential for future research.

The impact of cognition on perception warrants further attention and provides a fruitful arena for future research. We specifically focused on the cognitive impact of the extrinsic cue of advertising on sensory perception, but thoughts generated by other extrinsic cues could be equally as intriguing. Relevant neuroscience literature has shown that verbal labels of smells (e.g., cheddar cheese or body odor) affects the perception of the smell itself by inhibiting activation of smell areas in the brain for unpleasant smells (de Araujo et al. 2005). Should these processes occur in a more deliberative manner, as shown across our studies, we may gain valuable insight into the effects of cognitions on perceptions through behavioral methodologies as well. Further, in our studies the stimuli used were generally pleasant, leading to congruence between the advertisement and consumption experience. However, should there be marked incongruity between the extrinsic cue and the consumption experience, one could explore the potential dominance of cognitions over sensory perceptions or vice versa. Our cognitions shape our experiences in a top-down manner, but the bottom-up influences of perceptions obviously play a role as well. This interaction of both cognition and sensory perception warrants future attention.

With our research we chose to focus on the deliberate, cognitive determinants of sensory perceptions; however, future research could also address ways to affect these perceptions below consciousness, as much of what we do and perceive is driven by automatic processes (Bargh and Chartrand 1999). For example, it is possible that visual stimuli are processed more automatically than verbal stimuli in an advertising context, and this could affect taste perceptions even under cognitive constraint. Indeed, pictures of food lead to similar neural activation patterns in the orbitofrontal cortex as verbal descriptions, and additionally they activate areas associated with reward (Simmons, Martin, and Barsalou 2005). Therefore, it would be worthwhile to explore the impact of pictures used in isolation of verbal framing on sensory perceptions.

Managerial implications of this research follow directly from our results. Despite the conventional wisdom that taste is composed of multiple sensory inputs, advertising within the food and beverage industry rarely addresses perceptions beyond taste. The results from our studies suggest that advertising should include multiple sensory attributes of the products as this has a significant impact on perceptions of the product. These findings are particularly relevant for the food industry, including packaged goods and restaurants, as it continues to spend billions of dollars in advertising the taste of food, one of our most pleasurable and sensory experiences.

\section{REFERENCES}

Advertising Age Data Center (2008), http://adage.com/datacenter/ article?article_id $=127915 \&$ search_phrase $=$ top $\% 20$ spenders. Allison, Ralph I. and Kenneth P. Uhl (1964), "Influence of Beer 
Brand Identification on Taste Perception," Journal of Marketing Research, 1 (August), 36-39.

Bargh, John A. and Tanya L. Chartrand (1999), "The Unbearable Automaticity of Being," American Psychologist, 54 (July), 462-79.

Baron, Reuben M. and David A. Kenny (1986), "The ModeratorMediator Variable Distinction in Social Psychological Research: Conceptual, Strategic, and Statistical Considerations," Journal of Personality and Social Psychology, 51 (6), 1173-82.

Cacioppo, John T. and Richard E. Petty (1981), "Social Psychological Procedures for Cognitive Response Assessment: The Thought-Listing Technique," in Cognitive Assessment, ed. Thomas V. Merluzzi, Carol R. Glass, and Myles Genest, New York: Guilford, 309-42.

Chaudhari, Nirupa, Ana Marie Landin, and Stephen D. Roper (2000), "A Metabotropic Glutamate Receptor Variant Functions as a Taste Receptor," Nature Neuroscience, 3 (2), 113-19.

Cruz, Alberto and Barry G. Green (2000), "Thermal Stimulation of Taste," Nature, 403 (6772), 889-92.

de Araujo, Ivan E. and Edmund T. Rolls (2004), "Representation in the Human Brain of Food Texture and Oral Fat," Journal of Neuroscience, 24(12), 3086-93.

de Araujo, Ivan E., Edmund T. Rolls, Maria Ines Velazco, Christian Margot, and Isabelle Cayeux (2005), "Cognitive Modulation of Olfactory Processing," Neuron, 46 (4), 671-79.

DuBose, Cynthia N., Armand V. Cardello, and Owen Maller (1980), "Effects of Colorants and Flavorants on Identification, Perceived Flavor Intensity, and Hedonic Quality of Fruit-Flavored Beverages and Cake," Journal of Food Science, 45 (5), 1393-99.

Edell, Julie A. and Richard Staelin (1983), "The Information Processing of Pictures in Print Advertisements," Journal of Consumer Research, 10 (June), 45-61.

Gilbert, Daniel T., R. Brian Giesler, and Kathryn A. Morris (1995), "When Comparisons Arise," Journal of Personality and Social Psychology, 69 (August), 227-36.

Gilbert, Daniel T. and J. Gregory Hixon (1991), “The Trouble of Thinking: Activation and Application of Stereotypic Beliefs," Journal of Personality and Social Psychology, 60 (April), 509-17.

Herz, Rachel (2007), The Scent of Desire: Discovering Our Enigmatic Sense of Smell, New York: William Morrow.

Hoch, Stephen J. and Young-Won Ha (1986), “Consumer Learning: Advertising and the Ambiguity of Product Experience," Journal of Consumer Research, 13 (September), 221-33.

Hoegg, Joandrea and Joseph W. Alba (2007), "Taste Perception: More than Meets the Tongue," Journal of Consumer Research, 33 (March), 490-98.

Ikeda, Kikunae (2002), "New Seasonings," Chemical Senses, 27 (9), 847-49.

Krishna, Aradhna (2007), "Spatial Perception Research: An Integrative Review of Length, Area, Volume, and Number Perception," in Visual Marketing: From Attention to Action, ed. Michel Wedel and Rik Peters, Marketing and Consumer Psychology Series, New York: Erlbaum, 167-92.

Krishna, Aradhna and Maureen Morrin (2008), "Does Touch Affect
Taste? The Perceptual Transfer of Product Container Haptic Cues," Journal of Consumer Research, 34 (April), 807-18.

Lee, Leonard, Shane Frederick, and Dan Ariely (2006), "Try It, You'll Like It: The Influence of Expectation, Consumption, and Revelation on Preferences for Beer," Psychological Science, 17 (12), 1054-58.

Levin, Irwin P. and Gary J. Gaeth (1988), "How Consumers Are Affected by the Framing of Attribute Information before and after Consuming the Product," Journal of Consumer Research, 15 (December), 374-78.

Mitchell, Andrew A. and Jerry C. Olson (1981), "Are Product Attribute Beliefs the Only Mediator of Advertising Effects on Brand Attitude?" Journal of Marketing Research, 18 (August), 318-32.

Nowlis, Stephen M. and Baba Shiv (2005), "The Influence of Consumer Distractions on the Effectiveness of Food-Sampling Programs," Journal of Marketing Research, 42 (May), 157-68.

Peck, Joann and Terry L. Childers (2008), "If It Tastes, Smells, Sounds, and Feels Like a Duck, Then It Must Be a . . . : Effects of Sensory Factors on Consumer Behaviors," in Handbook of Consumer Psychology, ed. Curtis P. Haugtvedt, Paul M. Herr, and Frank R. Kardes, New York: Psychology, 193-219.

Raghunathan, Rajagopal, Rebecca W. Naylor, and Wayne D. Hoyer (2006), "The Unhealthy = Tasty Intuition and Its Effects on Taste Inferences, Enjoyment, and Choice of Food Products," Journal of Marketing, 70 (October), 170-84.

Rolls, Edmund T. (2005), "Taste, Olfactory, and Food Texture Processing in the Brain and the Control of Food Intake," Physiology and Behavior, 85 (May), 45-56.

Rozin, Paul (1982), "Taste-Smell Confusions and the Duality of the Olfactory Sense," Perception and Psychophysics, 31 (April), 397-401.

Shiv, Baba and Stephen M. Nowlis (2004), "The Effect of Distractions While Tasting a Food Sample: The Interplay of Informational and Affective Components in Subsequent Choice," Journal of Consumer Research, 31 (December), 599-608.

Simmons, W. Kyle, Alex Martin, and Lawrence Barsalou (2005), "Pictures of Appetizing Foods Activate Gustatory Cortices for Taste and Reward," Cerebral Cortex, 15 (10), 1602-8.

Small, Dana M. and Marilyn K. Jones-Gotman (2001), "Neural Substrates of Taste/Smell Interaction and Flavor in the Human Brain," Chemical Senses, 26 (8), 1034.

Small, Dana M. and John Prescott (2005), "Odor/Taste Integration and the Perception of Flavor," Experimental Brain Research, 166 (October), 345-57.

Smith, Edward E. and Stephen M. Kosslyn (2007), Cognitive Psychology: Mind and Brain, Upper Saddle River, NJ: Pearson Prentice Hall.

Sobel, Michael E. (1982), "Asymptotic Intervals for Indirect Effects in Structural Equations Models," in Sociological Methodology, ed. Samuel Leinhart, San Francisco: Jossey-Bass, 290-312.

Zampini, Massimiliano and Charles Spence (2004), "The Role of Auditory Cues in Modulating the Perceived Crispiness and Staleness of Potato Chips," Journal of Sensory Studies, 19 (5), 347-63. 